Figure 1. (A) OR for changes in FL prevalence vs presence or absence of inflammation at BL; (B) OR of FL for VEs with vs without inflammation at Week 12
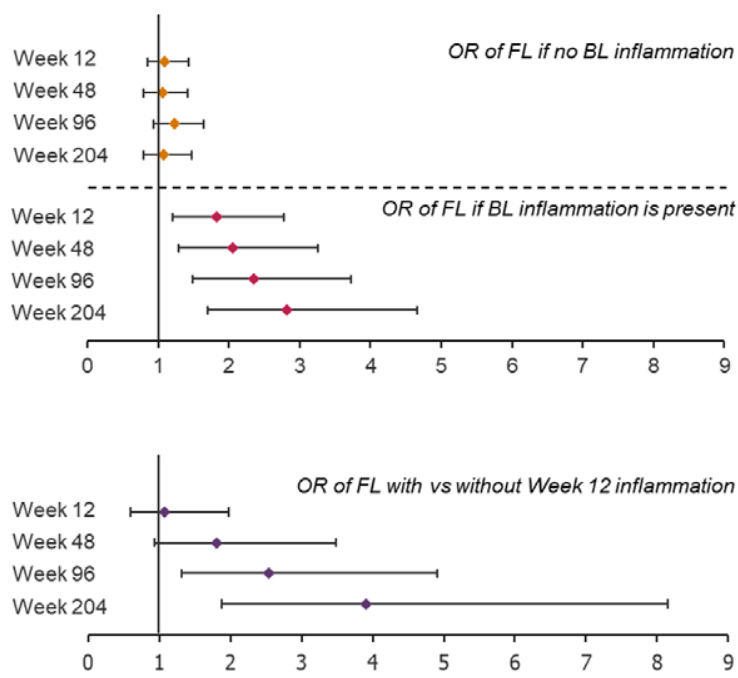

$A: A n O R>1$ represents an increase in $F L$ from $B L$. A logistic regression with fixed effects for $B L$ inflammation status by visit (including $B L$ ) and random effects for patient and $V E$ (within patient). $B: A n O R>1$ represents increased FL in VEs with vs without inflammation at Week 12, adjusted for BL VE status with respect to inflammation and FL. A logistic regression with fixed effects for Week 12 inflammation by visit (excluding BL) and BL inflammation by BL FL, and random effects for patient and VE (within patient). BL: baseline; FL: fatty lesion; OR: odds ratio; VE: vertebral edge.

Acknowledgments: This study was funded by UCB Pharma. Editorial services were provided by Costello Medical.

Disclosure of Interests: Xenofon Baraliakos Grant/research support from: Grant/ research support from: AbbVie, BMS, Celgene, Chugai, Merck, Novartis, Pfizer, UCB and Werfen, Consultant of: AbbVie, BMS, Celgene, Chugai, Merck, Novartis, Pfizer, UCB and Werfen, Speakers bureau: AbbVie, BMS, Celgene, Chugai, Merck, Novartis, Pfizer, UCB and Werfen, Sebastian Kruse: None declared, Simone Auteri Shareholder of: UCB Pharma, Employee of: UCB Pharma, Natasha de Peyrecave Employee of: UCB Pharma, Tommi Nurminen Employee of: UCB Pharma, Thomas Kumke Employee of: UCB Pharma, Bengt Hoepken Employee of: UCB Pharma, Juergen Braun Grant/research support from: Abbvie (Abbott), Amgen, BMS, Boehringer, Celgene, Celltrion, Centocor, Chugai, Eli Lilly and Company, Medac, MSD (Schering Plough), Mundipharma, Novartis, Pfizer (Wyeth), Roche, Sanofi- Aventis, and UCB Pharma, Consultant of: Abbvie (Abbott), Amgen, BMS, Boehringer, Celgene, Celltrion, Centocor, Chugai, EBEWE Pharma, Eli Lilly and Company, Medac, MSD (Schering-Plough), Mundipharma, Novartis, Pfizer (Wyeth), Roche, Sanofi-Aventis, and UCB Pharma, Speakers bureau: Abbvie (Abbott), Amgen, BMS, Boehringer, Celgene, Celltrion, Centocor, Chugai, EBEWE Pharma, Eli Lilly and Company, Medac, MSD (Schering-Plough), Mundipharma, Novartis, Pfizer (Wyeth), Roche, Sanofi-Aventis, and UCB Pharma

DOI: 10.1136/annrheumdis-2020-eular.4166

\section{OP0105 \\ EFFICACY AND SAFETY OF BIMEKIZUMAB IN ANKYLOSING SPONDYLITIS: 48-WEEK PATIENT- REPORTED OUTCOMES FROM A PHASE 2B, RANDOMISED, DOUBLE-BLIND, PLACEBO- CONTROLLED, DOSE-RANGING STUDY}

D. Van der Heijde ${ }^{1}$, L. S. Gensler ${ }^{2}$, A. Deodhar ${ }^{3}$, X. Baraliakos ${ }^{4}$, D. Poddubnyy ${ }^{5}$ A. Kivitz ${ }^{6}$, M. K. Farmer ${ }^{7}$, D. Baeten ${ }^{8}$, N. Goldammer ${ }^{9}$, J. Coarse ${ }^{7}$,

M. Oortgiesen ${ }^{7}$, M. Dougados ${ }^{10,11} .{ }^{1}$ Leiden University Medical Center, Leiden, Netherlands; ${ }^{2}$ University of California San Francisco, San Francisco, United States of America; ${ }^{3}$ Oregon Health \& Science University, Portland, United States of America; ${ }^{4}$ Rheumazentrum Ruhrgebiet, Herne, Germany; ${ }^{5}$ Charite - Universitätsmedizin Berlin, German Rheumatism Research Centre, Berlin, Germany; ${ }^{6}$ Altoona Center for Clinical Research, Duncansville, United States of America; ${ }^{7}$ UCB Pharma, Raleigh, United States of America; ${ }^{8}$ UCB Pharma, Slough, United Kingdom; ${ }^{9}$ UCB Pharma, Monheim am Rhein, Germany; ${ }^{10}$ Université de Paris, Department of Rheumatology, Paris, France; ${ }^{11}$ INSERM (U1153): Epidémiologie Clinique et Biostatistiques, PRES Sorbonne Paris-Cité, Paris, France

Background: Bimekizumab (BKZ), a monoclonal antibody that selectively neutralises interleukin (IL)-17A and IL-17F, is a potential therapeutic option in ankylosing spondylitis (AS).
Objectives: To report 48-week (wk) patient-reported outcomes (PROs) in patients (pts) with AS treated with $B K Z$ in a phase $2 b$ dose-ranging study (BE-AGILE; NCT02963506).

Methods: Pts with active AS (Bath AS Disease Activity Index [BASDAI] $\geq 4$; spinal pain $\geq 4[0-10]$ ), fulfilling modified New York criteria (central reading), and inadequate response/intolerance to NSAIDs were randomised according to the study design (Figure 1). PROs included spinal pain, fatigue (BASDAI Q1), morning stiffness (mean of BASDAI Q5+6), Bath AS Functional Index (BASFI), Medical Outcomes Study (MOS) Sleep Problems Index II and AS Quality of Life questionnaire (ASQoL). Efficacy is reported for pts initially randomised to placebo (PBO) or BKZ 160/320 mg every 4 weeks (Q4W); treatment-emergent adverse events (TEAEs) are reported for pts who received $\geq 1$ dose of study drug (Safety Set). Results: Of 303 pts, 181 were randomised to PBO or BKZ 160/320 Q4W mg at Wk 0;179/181 completed Wk 12 and 161/181 completed Wk 48. At Wk 12 improvements in pain, fatigue, morning stiffness, BASFI, sleep and ASQoL were greater in BKZ pts vs $\mathrm{PBO}$ pts. Responses were further improved or maintained to Wk 48, with no meaningful differences between BKZ $160 \mathrm{mg}$ and $320 \mathrm{mg}$ (Table 1). Serious TEAEs occurred in $13 / 303$ (4.3\%) pts (Table 2), which included 2 major adverse cardiac events considered not related to study drug. Oral candidiasis occurred in 16 (5.3\%) pts.

Figure 1. Study design

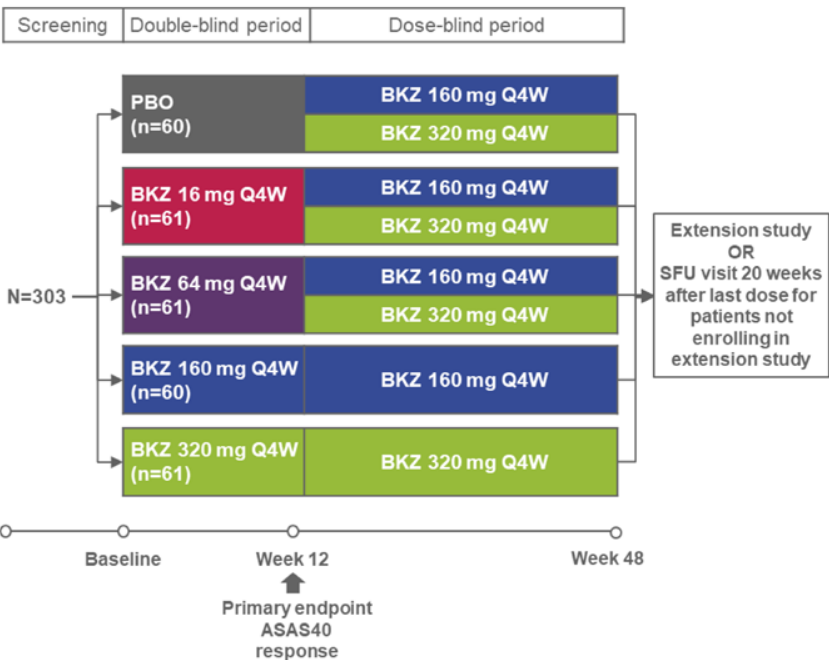

ASAS40: Assessment of SpondyloArthritis international Society improvement of $\geq 40 \%$; Q4W: every 4 weeks; SFU: safety follow-up.

Table 1. PRO efficacy endpoints to Week 48 (multiple imputation)

\begin{tabular}{lccccc}
\hline Mean (SD) & Wk & $\begin{array}{c}\text { PBO }-\mathrm{BKZ} \\
160 \mathrm{mg} \\
(\mathrm{n}=24)\end{array}$ & $\begin{array}{c}\text { PBO }-\mathrm{BKZ} \\
320 \mathrm{mg} \\
(\mathrm{n}=36)\end{array}$ & $\begin{array}{c}\mathrm{BKZ} 160 \mathrm{mg} \\
(\mathrm{n}=58)\end{array}$ & $\begin{array}{c}\mathrm{BKZ} \\
320 \mathrm{mg} \\
(\mathrm{n}=61)\end{array}$ \\
\hline Spinal pain & 0 & $6.9(1.4)$ & $7.0(1.9)$ & $6.6(2.0)$ & $7.3(1.5)$ \\
CfB & 12 & $-1.5(1.6)$ & $-0.7(1.7)$ & $-2.6(2.2)$ & $-3.6(2.4)$ \\
& 48 & $-3.7(2.0)$ & $-3.7(2.6)$ & $-3.8(2.4)$ & $-4.7(2.1)$ \\
Fatigue & 0 & $6.4(1.7)$ & $6.8(1.6)$ & $6.4(1.7)$ & $6.4(1.9)$ \\
CfB & 12 & $-0.7(2.5)$ & $-1.0(1.7)$ & $-2.1(2.2)$ & $-2.1(2.5)$ \\
& 48 & $-2.7(2.2)$ & $-2.8(2.4)$ & $-3.1(2.1)$ & $-3.3(2.4)$ \\
Morning stiffness & 0 & $6.9(1.7)$ & $6.7(2.0)$ & $6.5(1.8)$ & $6.6(2.1)$ \\
CfB & 12 & $-1.5(1.7)$ & $-1.1(1.5)$ & $-2.8(2.0)$ & $-3.4(2.7)$ \\
& 48 & $-3.9(2.2)$ & $-3.6(2.4)$ & $-3.9(2.2)$ & $-4.4(2.4)$ \\
BASFI & 0 & $5.8(1.8)$ & $5.5(2.2)$ & $5.5(2.2)$ & $5.9(2.0)$ \\
CfB & 12 & $-1.0(2.1)$ & $-0.3(1.7)$ & $-1.7(1.8)$ & $-2.2(2.0)$ \\
& 48 & $-2.9(2.2)$ & $-2.4(2.2)$ & $-2.5(2.0)$ & $-2.9(2.2)$ \\
MOS Sleep Problems & 0 & $45.5(8.1)$ & $45.3(7.9)$ & $46.9(7.5)$ & $47.2(9.4)$ \\
Index II & & & & & \\
CfB & 12 & $2.1(8.3)$ & $1.8(6.8)$ & $5.6(6.7)$ & $6.8(7.5)$ \\
& 48 & $7.6(8.7)$ & $8.0(9.1)$ & $6.5(6.1)$ & $8.0(7.9)$ \\
ASQoL & 0 & $8.4(4.7)$ & $9.2(4.7)$ & $8.4(4.3)$ & $8.7(4.3)$ \\
CfB & 12 & $-1.3(5.5)$ & $-1.3(3.7)$ & $-3.5(4.3)$ & $-4.6(4.8)$ \\
& 48 & $-4.2(5.6)$ & $-5.3(5.6)$ & $-4.9(4.1)$ & $-5.4(4.8)$ \\
& & & & &
\end{tabular}

CfB: change from baseline

Conclusion: Pts with active AS demonstrated rapid and sustained improvements in PROs, sleep and quality of life over 48 wks of BKZ treatment. BKZ was generally well tolerated with no unexpected safety findings versus previous studies.

Acknowledgments: This study was funded by UCB Pharma. Editorial services were provided by Costello Medical. 
Table 2. Overview of TEAEs to Week 48 (Safety Set; $N=303$ )

\begin{tabular}{lccc}
\hline $\mathrm{n}(\%)$ & $\begin{array}{c}\text { BKZ 160 mg } \\
(\mathrm{n}=149)\end{array}$ & $\begin{array}{c}\text { BKZ 320 mg } \\
(\mathrm{n}=150)\end{array}$ & $\begin{array}{c}\text { All BKZ [a] } \\
(\mathrm{N}=303)\end{array}$ \\
\hline Any TEAE & $103(69.1)$ & $122(81.3)$ & $235(77.6)$ \\
Drug-related TEAEs & $48(32.2)$ & $54(36.0)$ & $110(36.3)$ \\
Serious TEAEs & $5(3.4)$ & $6(4.0)$ & $13(4.3)$ \\
Discontinuations due to TEAEs & $7(4.7)$ & $10(6.7)$ & $20(6.6)$ \\
\hline
\end{tabular}

[a] Includes TEAEs for 16 and $64 \mathrm{mg} \mathrm{BKZ}$

Disclosure of Interests: Désirée van der Heijde Consultant of: AbbVie, Amgen, Astellas, AstraZeneca, BMS, Boehringer Ingelheim, Celgene, Cyxone, Daiichi, Eisai, Eli-Lilly, Galapagos, Gilead Sciences, Inc., Glaxo-Smith-Kline, Janssen, Merck, Novartis, Pfizer, Regeneron, Roche, Sanofi, Takeda, UCB Pharma; Director of Imaging Rheumatology BV, Lianne S. Gensler Grant/research support from: Pfizer, Novartis, UCB, Consultant of: AbbVie, Eli Lilly, GSK, Novartis, UCB, Atul Deodhar Grant/research support from: AbbVie, Eli Lilly, GSK, Novartis, Pfizer, UCB, Consultant of: AbbVie, Amgen, Boehringer Ingelheim, Bristol Myer Squibb (BMS), Eli Lilly, GSK, Janssen, Novartis, Pfizer, UCB, Speakers bureau: AbbVie, Amgen, Boehringer Ingelheim, Bristol Myer Squibb (BMS), Eli Lilly, GSK, Janssen, Novartis, Pfizer, UCB, Xenofon Baraliakos Grant/research support from: Grant/research support from: AbbVie, BMS, Celgene, Chugai, Merck, Novartis, Pfizer, UCB and Werfen, Consultant of: AbbVie, BMS, Celgene, Chugai, Merck, Novartis, Pfizer, UCB and Werfen, Speakers bureau: AbbVie, BMS, Celgene, Chugai, Merck, Novartis, Pfizer, UCB and Werfen, Denis Poddubnyy Grant/research support from: AbbVie, MSD, Novartis, and Pfizer, Consultant of: AbbVie, Bristol-Myers Squibb, Eli Lilly, MSD, Novartis, Pfizer, Roche, UCB, Speakers bureau: AbbVie, Bristol-Myers Squibb, Eli Lilly, MSD, Novartis, Pfizer, Roche, UCB, Alan Kivitz Shareholder of: AbbVie, Amgen, Gilead, GSK, Pfizer Inc, Sanofi, Consultant of: AbbVie, Boehringer Ingelheim, Flexion, Genzyme, Gilead, Janssen, Novartis, Pfizer Inc, Regeneron, Sanofi, SUN Pharma Advanced Research, UCB, Paid instructor for: Celgene, Genzyme, Horizon, Merck, Novartis, Pfizer, Regeneron, Sanofi, Speakers bureau: AbbVie, Celgene, Flexion, Genzyme, Horizon, Merck, Novartis, Pfizer Inc, Regeneron, Sanofi, Mary Katherine Farmer Employee of: UCB Pharma, Dominique Baeten Employee of: UCB Pharma, Nadine Goldammer Employee of: UCB Pharma, Jason Coarse Employee of: UCB Pharma, Marga Oortgiesen Employee of: UCB Pharma, Maxime Dougados Grant/research support from: AbbVie, Eli Lilly, Merck, Novartis, Pfizer and UCB Pharma, Consultant of: AbbVie, Eli Lilly, Merck, Novartis, Pfizer and UCB Pharma, Speakers bureau: AbbVie, Eli Lilly, Merck, Novartis, Pfizer and UCB Pharma

DOI: 10.1136/annrheumdis-2020-eular.323

\section{OP0106 \\ SECUKINUMAB 150 MG SIGNIFICANTLY IMPROVED SIGNS AND SYMPTOMS OF NON-RADIOGRAPHIC AXIAL SPONDYLOARTHRITIS: 52-WEEK RESULTS FROM THE PHASE III PREVENT STUDY}

J. Braun ${ }^{1}$, R. Blanco ${ }^{2}$, E. Dokoupilova ${ }^{3}$, L. S. Gensler ${ }^{4}$, A. Kivitz ${ }^{5}$, S. Hall ${ }^{6}$, H. Kameda ${ }^{7}$, D. Poddubnyy ${ }^{8}$, M. Van de Sande ${ }^{9}$, D. Van der Heijde ${ }^{10}$, A. Wiksten ${ }^{11}$, B. Porter ${ }^{12}$, H. Richards ${ }^{11}$, S. Haemmerle ${ }^{11}$, A. Deodhar ${ }^{13} .{ }^{1}$ Ruhr Univ. Bochum, Bochum, Germany; ${ }^{2}$ Hospital Univ. Marqués de Valdecilla, Santander, Spain; ${ }^{3}$ MEDICAL PLUS s.r.o., Univ. of Veterinary \& Pharma. Sciences, Brno, Czech Republic; ${ }^{4}$ Univ. of California, San Francisco, San Francisco, United States of America; ${ }^{5}$ Altoona Center for Clinical Research, Duncansville, United States of America; ${ }^{6}$ Monash Univ., Melbourne, Australia; ${ }^{7}$ Toho Univ., Tokyo, Japan; ${ }^{8}$ Charité Universitätsmedizin Berlin, Berlin, Germany; ${ }^{9}$ Rheumatology \& Immunology Center, Amsterdam, Netherlands; ${ }^{10}$ Univ. Medical Centre, Leiden, Netherlands; ${ }^{11}$ Novartis Pharma AG, Basel, Switzerland; ${ }^{12}$ Novartis Pharma. Corp., East Hanover, United States of America; ${ }^{13}$ Oregon Health \& Science Univ., Portland, United States of America

Background: Axial spondyloarthritis (axSpA) spectrum covers radiographic axSpA and non-radiographic axSpA (nr-axSpA). PREVENT (NCT02696031) is the first phase III, placebo (PBO) controlled study evaluating secukinumab (SEC) $150 \mathrm{mg}$ with (LD) or without loading (NL) dose, in patients (pts) with nr-axSpA. ${ }^{1}$ The study had 2 independent analysis plans as per EU (Wk 16) and US (Wk 52) regulatory requirements.

Objectives: To report efficacy through Wk 52 and safety up to two years for the PREVENT study.

Methods: 555 pts fulfilling ASAS criteria for axSpA plus abnormal CRP and/or $\mathrm{MRI}$, without evidence of radiographic changes in sacroiliac (SI) joints according to modified New York Criteria for AS were enrolled. All images were assessed centrally before inclusion. Pts were randomised $(1: 1: 1)$ to SEC $150 \mathrm{mg}$ with LD, $\mathrm{NL}$, or PBO at baseline (BL). LD pts received SEC $150 \mathrm{mg}$ at Wks 1, 2, 3, and 4 , and then every 4 wks (q4wk) starting at Wk 4. NL pts received SEC $150 \mathrm{mg}$ at $\mathrm{BL}$ and PBO at Wks 1, 2, and 3, and then $150 \mathrm{mg}$ q4wk. Switch to open-label
(OL) SEC $150 \mathrm{mg}$ or standard of care (SoC) was permitted after Wk 20. Primary endpoint was ASAS40 at Wk 16 (LD) and at Wk 52 (NL) in anti-TNF-naïve pts. Secondary endpoints (overall population) included ASAS40, BASDAI50, SI joint bone marrow edema (BME) score by MRI at Wks 16 and 52 and ASDAS-CRP inactive disease (ID) at Wk 52. Endpoints were analysed according to statistical hierarchy. Analysis used non responder imputation through Wk 52 . Safety analyses included all pts who received $\geq 1$ dose of study treatment.

Results: Overall, 481 pts completed 52 wks with no major differences in retention across groups: 84.3\% (156/185; LD), 89.7\% (165/184; NL) and 86.0\% (160/186; PBO). BL characteristics were similar across groups; $90 \%$ pts were anti-TNFnaïve, $56-58 \%$ pts had elevated CRP, $71-75 \%$ pts had evidence of SI joint inflammation by MRI. Proportion of pts who switched to OL or SoC between Wks 20 and 48 was $52.1 \%$ (LD), $49.2 \%$ (NL), and $67.4 \%$ (PBO). Primary endpoints at Wk 16 and Wk 52 were met (Table). SEC $150 \mathrm{mg}$ LD or NL significantly improved secondary endpoints at Wk 16 and 52 vs $\mathrm{PBO}$ (Table). SEC significantly reduced SI joint MRI BME score vs PBO at WK $16(-1.68$ and -1.03 vs $-0.39 ; P=0.0197$ and 0.026 , LD and NL respectively). No unexpected safety signals were reported. Conclusion: SEC $150 \mathrm{mg}$ provided significant and sustained improvement in signs and symptoms of pts with nr-axSpA through Wk 52. MRI BME scores were reduced accordingly. There was no major difference between LD and NL. Safety of SEC was consistent with previous reports. ${ }^{2}$

References:

[1] Deodhar A, et al. Arthritis Rheumatol. 2019;71(suppl 10).

[2] Deodhar A, et al. Arth Res Ther. 2019;21:111.

\section{Table}

\begin{tabular}{lcccc}
\hline Endpoints, \% responders & Wk & $\begin{array}{c}\text { SEC } \\
150 \mathrm{mg} \text { LD } \\
(\mathrm{N}=185)\end{array}$ & $\begin{array}{c}\text { SEC } \\
150 \mathrm{mg} \mathrm{NL} \\
(\mathrm{N}=184)\end{array}$ & $\begin{array}{c}\text { PBO } \\
(\mathrm{N}=186)\end{array}$ \\
\hline Primary & & & & \\
ASAS40 in anti-TNF-naïve pts & 16 & $41.5^{\ddagger}$ & $42.2^{\ddagger}$ & 29.2 \\
& 52 & $35.4^{\ddagger}$ & $39.8^{\ddagger}$ & 19.9 \\
Secondary & 16 & $40.0^{\ddagger}$ & $40.8^{\ddagger}$ & 28.0 \\
ASAS40 & 52 & $33.5^{\ddagger}$ & $38.0^{\ddagger}$ & 19.4 \\
BASDAI50 & 16 & $37.3^{\ddagger}$ & $37.5^{\ddagger}$ & 21.0 \\
& 52 & $30.8^{\ddagger}$ & $35.3^{\ddagger}$ & 19.9 \\
ASDAS-CRP ID & 16 & $20.5^{\dagger}$ & $21.7^{\dagger}$ & 8.1 \\
& 52 & 15.7 & $23.9^{\ddagger}$ & 10.2
\end{tabular}

${ }^{\dagger} P<0.001 ;{ }^{\ddagger} P<0.05$ vs PBO ( $P$ values are adjusted for multiplicity of testing at Wks 16 and 52. Unadjusted $P$ value for ASDAS-CRP ID at Wk 16). Missing values were imputed as non-response.

$\mathrm{N}$, number of randomised pts

Disclosure of Interests: Juergen Braun Grant/research support from: Abbvie (Abbott), Amgen, BMS, Boehringer, Celgene, Celltrion, Centocor, Chugai, El Lilly and Company, Medac, MSD (Schering Plough), Mundipharma, Novartis, Pfizer (Wyeth), Roche, Sanofi- Aventis, and UCB Pharma, Consultant of: Abbvie (Abbott), Amgen, BMS, Boehringer, Celgene, Celltrion, Centocor, Chugai, EBEWE Pharma, Eli Lilly and Company, Medac, MSD (Schering-Plough), Mundipharma, Novartis, Pfizer (Wyeth), Roche, Sanofi-Aventis, and UCB Pharma, Speakers bureau: Abbvie (Abbott), Amgen, BMS, Boehringer, Celgene, Celltrion, Centocor, Chugai, EBEWE Pharma, Eli Lilly and Company, Medac, MSD (Schering-Plough), Mundipharma, Novartis, Pfizer (Wyeth), Roche, SanofiAventis, and UCB Pharma, Ricardo Blanco Grant/research support from: AbbVie, MSD, Roche, Consultant of: Abbvie, Eli Lilly, Pfizer, Roche, Bristol-Myers, Janssen, UCB Pharma and MSD, Speakers bureau: Abbvie, Eli Lilly, Pfizer Roche, Bristol-Myers, Janssen, UCB Pharma. MSD, Eva Dokoupilova Grant/ research support from: Eli Lilly, AbbVie, Novartis, Lianne S. Gensler Grant/ research support from: Pfizer, Novartis, UCB, Consultant of: AbbVie, Eli Lilly, GSK, Novartis, UCB, Alan Kivitz Shareholder of: AbbVie, Amgen, Gilead, GSK, Pfizer Inc, Sanofi, Consultant of: AbbVie, Boehringer Ingelheim, Flexion, Genzyme, Gilead, Janssen, Novartis, Pfizer Inc, Regeneron, Sanofi, SUN Pharma Advanced Research, UCB, Paid instructor for: Celgene, Genzyme, Horizon, Merck, Novartis, Pfizer, Regeneron, Sanofi, Speakers bureau: AbbVie, Celgene, Flexion, Genzyme, Horizon, Merck, Novartis, Pfizer Inc, Regeneron, Sanofi, Stephen Hall Grant/research support from: Abbvie, UCB, Janssen, Merck, Hideto Kameda Grant/research support from: Abbvie, Asahi-Kasei, Chugai, Eisai, Mitsubishi-Tanabe and Novartis, Consultant of: Abbvie, Boehringer, Celgene, Eli Lilly, Janssen, Novartis, Sanofi, UCB, Speakers bureau: Abbvie, Asahi-Kasei, BMS, Chugai, Eisai, Eli Lilly, Janssen, Mitsubishi-Tanabe, Novartis and Pfizer, Denis Poddubnyy Grant/research support from: AbbVie, MSD, Novartis, and Pfizer, Consultant of: AbbVie, Bristol-Myers Squibb, Eli Lilly, MSD, Novartis, Pfizer, Roche, UCB, Speakers bureau: AbbVie, Bristol-Myers Squibb, Eli Lilly, MSD, Novartis, Pfizer, Roche, UCB, Marleen van de Sande Grant/research support from: Novartis, Eli Lilly, Boehringer Ingelheim, Janssen, Consultant of: Abbvie, Novartis, Eli Lilly, Speakers bureau: Novartis, MSD, Désirée van der Heijde 